\title{
EDITOR'S

\section{Best Videos of the Year 2012: Awards Recognizing Contributions to our Field}

I would like to firstly thank the current editor-in-chief of the International Brazilian Journal of Urology Dr. Sidney Glina for his strong support of the video section and enthusiasm in the selection of the best videos of the year. I would like to thank as well Dr. Francisco Sampaio who was truly instrumental in the development of the video section several years ago when he served as the editor-in-chief of the International Brazilian Journal of Urology. As editor of the video section, I have truly been impressed by the exponential growth of the number of high quality video submissions sent to us for review over the past year. Genitourinary surgeons from Brazil, other Latin American countries, and across the world have continually raised the bar of what can be surgically approached using minimally invasive and other novel techniques which is truly revolutionizing our surgical field. These awards for best videos of 2012 are a recognition of these efforts and contributions.

The means by which the best videos of 2012 were selected is as follows. All videos for these awards must have been selected for publication in the International Brazilian Journal of Urology during 2012 (between January 1st, 2012 and December 31st, 2012). All of the editorial consultant board members of the video section of the International Brazilian Journal of Urology were then asked to select which they felt deserved the 1st, 2nd, and 3rd prizes for best videos of the year which was conducted using an electronic survey. Videos were judged based on: 1) the originality of their surgical approach, 2) scientific contribution in moving our specialty forward, and 3) overall quality of the surgical video. To avoid any biases in the review of these videos, editorial consultants were asked not to select videos they had themselves contributed to and that were being considered for videos of the year. This being said, it gives me great pride to announce the best videos of the year with a synopsis of the highlights of these videos and of their contributing author(s).

\section{First Prize}

The first prize was awarded to the video "Single port transvesical prostatectomy" by the group of Vicentini et al. (1) from the urology department at the University of Sao Paulo, Brazil. The authors would like to highlight that Dr. Hisano is going to present their experience at the European Congress of Urology in Milan this upcoming year. This paper is as well being prepared for submission as a comprehensive manuscript in the months to come. Dr. Hisano, Chief of the Laparoscopic Section of the Urology Department, has worked together with Dr. Vicentini since their first extra-peritoneoscopic surgery, and their surgical series is a tribute to hard team work in which a surgical approach/technique was developed through mutual efforts.

The authors would like to thank Dr. Agresta, Dr. Murta, and Professor Claro for all their help in developing this surgical approach, in addition to all colleagues, residents, and center directors at their institution, who imparted working conditions and support to enable the present work. 


\section{EDITOR'S \\ COMMENT}

In this surgical video, Vicentini and colleagues demonstrate their first case of single port transvesical prostatectomy. They embarked on developing the present surgical approach after a previous experience with extraperitoneoscopic transvesical prostatectomy, which they quickly realized had several pitfalls, as the extended time for the creation of an extraperitoneal space and in performing and closing the bladder cystostomy. The use of the present single port device resulted in relatively short surgical times, with a direct access to the bladder, using the same laparoscopic instrumentation, while imparting the same benefits of an extraperitoneoscopic surgical procedure, including maintaining minimal intra-operative blood losses. Their cumulative experience with this technique is excellent providing impetus for the authors to refine this technique for larger sized prostates. Despite this procedure representing a relatively simple surgical approach, it requires nevertheless experience with laparoscopic minimally invasive surgical techniques although remaining quite feasible.

\section{Second Prize}

The second prize was awarded to the video "Video-assisted left inguinal lymphadenectomy for penile cancer" by the group of Britto et al. (2) from the Federal University of Rio Grande do Norte (UFRN), Brazil. Dr Cesar Araujo Britto graduated from medical school at the UFRN in 1999 followed by completion of his general surgical and urological training at the UNIFESP-Paulista school of medicine. He has thereafter served as a professor of urology at the UFRN since 2009. In the present video, Dr. Britto and colleagues demonstrate the anatomy of the inguinal region as seen laparoendoscopically. This constitutes the first such surgical procedure performed within their urological department and their reasons for publishing this work is that no such surgical video can be found on the internet. Similarly, the authors realize the importance of watching surgical videos particularly when tackling such novel procedures. Although surgical descriptions and illustrations (diagrams and photos) can provide the initial tools to adopt new approaches, narrated surgical videos have far greater purpose to our readership which predominantly consists of urological surgeons.

\section{Third Prize}

The third prize was awarded to the video "Retroperitoneal lymph node dissection with concomitant IVC thrombectomy, caval wall resection, and grafting for metastatic NSGCT" by my group at the Moffitt Cancer Center and the University of South Florida (3). The first point to highlight is that as the editor of the video section, I abstained from selecting any videos I was affiliated with in the consideration for published videos of the year. That being said, our video depicts the very unusual case of teratoma in the context of testis cancer which is locally invasive into major vascular structures. In addition, in this specific case, the patient had developed an IVC tumor thrombus following vascular invasion by the teratomatous containing tumor. This clearly is a highly complex surgical clinical presentation requiring the concerted participation of a highly skilled and dedicated team of urologists, vascular surgeons, and anesthestists. The principles of proximal and distal vascular 


\section{EDITOR'S \\ COMMENT}

control are well depicted in this video as well brings about the often asked question as to when should a vascular graft be employed versus simple ligation of the infrarenal IVC? There is no simple answer to this question however in younger patients where collateral venous drainage is suspected to not have been well established due to the acute nature of the vascular occlusion, vascular grafting maybe a more suitable option. Clinical questions that remain unanswered are how long to these venous grafts typically stay patent and should such patients be initiated on full anticoagulation therapy (and for how long)? Hopefully, such clinical questions will be answered in the years to come which I suspect will originate from scientific contributions in the vascular surgical literature.

My colleagues and I practice at the Moffitt Cancer Center (an NCI designated cancer center) and Tampa General Hospital in Florida. I (myself) am a trained urologic oncologist (having completed an SUO accredited fellowship at the MD Anderson Cancer Center in Houston, Texas) with a busy academic and surgical practice focused on kidney, penile, bladder, and testis cancer. I have placed great emphasis on employing minimally invasive surgical techniques including robotics in my surgical management of genitourinary malignancies. I take great satisfaction in tackling complex surgical cases including high level IVC tumor thrombi and other major vascular and urinary reconstructions within the realm of my surgical practice.

I hope you will find this new concept of best videos of the year beneficial and I look forward to your contributions in the months to come. Lastly, I would like to take this opportunity to wish you and your families a very happy and healthy 2013 !

\section{REFERENCES}

1. Vicentini FC, Hisano M, Agresta TS, Murta CB, 3 . Claro JF: Single port transvesical prostatectomy. Int Braz J Urol. 2012; 38: 432; discussion 433.

2. Britto CA, Rebouças RB, Lopes TR, Silva da Costa T, Leite Rde C, de Carvalho PS: Videoassisted left inguinal lymphadenectomy for penile cancer. Int Braz J Urol. 2012; 38: 289; discussion 290.
3. Hakky T, Kim T, Rodriguez AR, Armstrong P, Mangar $D$, Spiess PE: Retroperitoneal lymph node dissection with concomitant IVC thrombectomy, caval wall resection, and grafting for metastatic NSGCT. Int Braz J Urol. 2012; 38: 135; discussion 136.

Dr. Philippe E. Spiess, MD, FACS, FRCS (C)

Department of Genitourinary Oncology Moffitt Cancer Center Video Section Editor International Braz J Urol 